\title{
Medical thoracoscopy without pleural fluid: How I do it
}

\author{
Mario Tamburrini ${ }^{1}$, Unnati Desai ${ }^{2}$, Giovanni Fanti ${ }^{3}$, Angelo Scarda ${ }^{3}$, Francesca Zampieri ${ }^{3}$, \\ Umberto Zuccon $^{3}$, Parikshit Thakare ${ }^{2}$, Marco Contoli ${ }^{1,4}$, Alberto Papi ${ }^{1,4}$ \\ ${ }^{1}$ Pneumology Unit, Arcispedale Sant'Anna, University Hospital of Ferrara, Italy; ${ }^{2}$ Department of Pulmonary Medicine, \\ TNMC \& BYL Nair Hospital, Mumbai, India; ${ }^{3}$ Friuli Venezia Giulia Health Authority, Pordenone, Italy; ${ }^{4}$ Respiratory \\ Medicine, University of Ferrara, Italy
}

\begin{abstract}
Thoracoscopy is commonly used minimally invasive procedure in the field of interventional pulmonology. While medical thoracoscopy is the widely preferred modality, modifications to the technique and expansion in the scope of its utility have always challenged the conventional approach. We describe a modified technique of medical thoracoscopy in absence of pleural effusion also known as dry thoracoscopy under sedation and local anaesthesia.
\end{abstract}

\section{Introduction}

The availability of pleuroscope and medical thoracoscopy has revolutionised work up and care of pleural effusions of varied aetiologies. Conventionally, medical thoracoscopy is performed in the

Correspondence: Dr. Mario Tamburrini, Interventional Pulmonologist, Pneumology Unit, Arcispedale Sant'Anna, University Hospital of Ferrara, Italy.

Tel. +39.349.0091667.

E-mail: mario.tamburrini@ospfe.it

Key words: Pleural effusion; pleuroscope; thoracoport.

Contributions: All the authors made a substantive intellectual contribution, read and approved the final version of the manuscript and agreed to be accountable for all aspects of the work.

Funding: The authors do not have specific grant for this research from any funding agency in the public, commercial or not-for-profit sectors.

Conflict of interest: The authors declare that they have no competing interests, and all authors confirm accuracy.

Received for publication: 21 April 2020.

Accepted for publication: 11 June 2021.

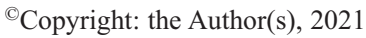

Licensee PAGEPress, Italy

Monaldi Archives for Chest Disease 2022; $92: 1331$

doi: 10.4081/monaldi.2021.1331

This article is distributed under the terms of the Creative Commons Attribution Noncommercial License (by-nc 4.0) which permits any noncommercial use, distribution, and reproduction in any medium, provided the original author(s) and source are credited. presence of pleural effusion. However, modifications to the said technique have been attempted to expand the scope of medical thoracoscopy in pleural malignancies which often have pleural nodularity with pleural thickening and minimal pleural effusion. This procedure of medical thoracoscopy in absence of pleural effusion also referred to as dry thoracoscopy has been described rarely and first by Marchetti. They however performed the procedure under general anaesthesia. We modified the procedure in lieu with the advances and availability of newer techniques, equipment and anaesthesia. We describe the unique procedure of dry thoracoscopy under local anaesthesia and sedation.

\section{Case Report}

A 76-year-old ex-smoker (30 pack years), diabetic, hypertensive with chronic kidney disease was referred with left chest pain, left minimal pleural effusion with pleural thickening which is confirmed on the chest X ray (CXR) and computed tomography (CT) of thorax. A positron emission tomography (PET) scan was performed in view of the non-tappable pleural effusion to determine further course of action which showed an uptake in the left lower thickened pleura (Figure 1). The patient was evaluated for the possibility of performing ultrasound percutaneous biopsies but the small size of the pleural lesions and the presence of the ribs made the procedure difficult to perform.

The patient was posted for medical thoracoscopy in absence of pleural effusion also known as dry thoracoscopy under local anaesthesia and moderate sedation in view of multiple comorbidities and high risk for general anaesthesia.

\section{Thoracoscopy procedure details}

The site was selected using clinico-radiological correlation in the safe triangle of the chest. The lung sliding sign was confirmed on ultrasound to determine the site of entry with the patient in the right lateral decubitus position. Parts were painted and draped. The procedure was performed under moderate sedation with the use of fentanyl, midazolam and propofol. Local anaesthesia was instilled at the site of thoracoport insertion. A $5 \mathrm{~mm}$ skin incision was taken at the site of entry. The $5 \mathrm{~mm}$ thoracoport of make Endopath eXcel $5 \mathrm{~mm}$ bladeless trocar with stability sleeve was inserted with the zerodegree camera lens in situ through the site with rotary action under camera vision till the white pleura was breached (Figures 2 and 3, Video 1). Following the entry in to the pleura, iatrogenic artificial 
pneumothorax was created by allowing the atmospheric air to enter the thoracic cavity. This caused collapse of the lung to allow further thoracoscopy procedure. On thoracoscopy the parietal pleura was visualised as diffusely thickened with whitish nodules in the mediobasal and diaphragmatic aspects with few nodules in the mediastinal pleura. Lung was diffusely anthracotic. Multiple biopsies (with $3 \mathrm{~mm}$ optic biopsy clamp strut) were performed on the thoracic pleural surface without obvious bleeding. Following procedure, talc pleurodesis was performed and an intercostal drain inserted through the entry site. The biopsy proved adenocarcinoma on histopathology.

\section{Discussion}

In 1882 coinciding with the discovery of the tubercle bacilli, Forlanini performed the first "pneumotorace artificiale" in tuber- culosis (TB). From 1910-1922, Hans Christian Jacobaeus performed the closed intrapleural pneumonolysis using a two canula technique labelling it as thoracoscopy since artificial pneumothorax prevented successful treatment in all TB patients. Hence he is known as the father of modern thoracoscopy. Following this Jacobeus technique was widely used as operative thoracoscopy till the advent of streptomycin in 1945. In the anti-TB drug era, the therapeutic need of thoracoscopy declined and biopsy remained the only indication. Video-assisted thoracoscopy (VATS) and the wide range of operating instruments and stapling devices developed again, brought thoracoscopy to the fore of thoracic surgery. Though this technique remained a surgeons fortay $[1,2]$.

Thoracoscopy is a diagnostic and therapeutic procedure for pleural pathologies. It is further classified into medical thoracoscopy and surgical thoracoscopy. Medical thoracoscopy is thoracoscopy performed under anaesthesia in the endoscopy suite performed mostly by a pulmonologist. In contrast, VATS is described as a key-

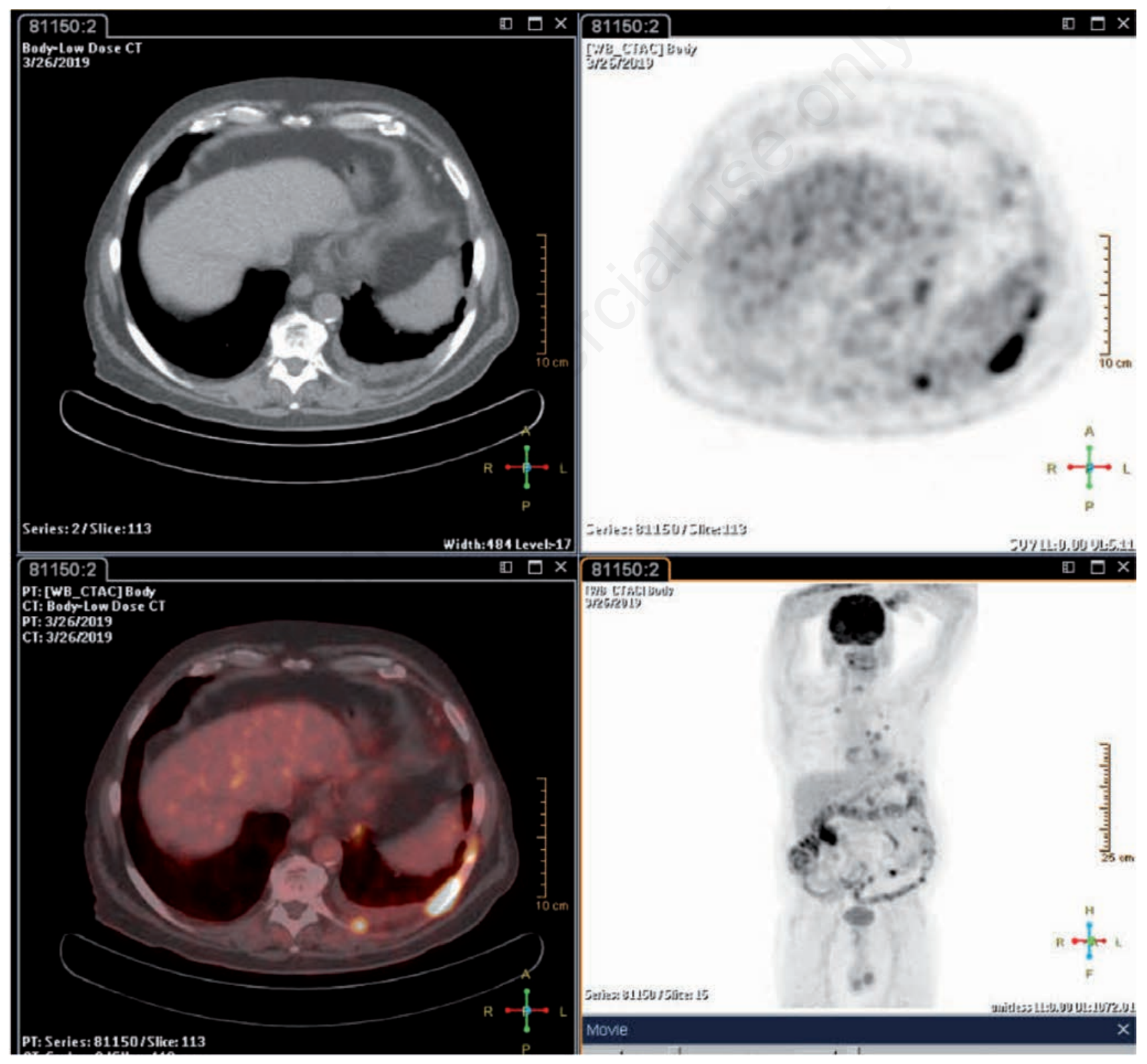

Figure 1. PET CT image. 
hole surgical procedure in the operating room, under general anaesthesia with one-lung ventilation performed mostly by a cardio-thoracic surgeon. Medical thoracoscopy was discovered to be minimally invasive alternative to cover the diagnostic aspects while surgical thoracoscopy was already available to cover the therapeutic aspects. The most frequent indication for diagnostic thoracoscopy is pleural effusion, pleural nodularity with effusion and rarely spontaneous pneumothorax to identify the cause of the pneumothorax. The most frequent indication for therapeutic thoracoscopy is pleurodesis (mostly chemical) followed by rarer indications like bullectomy, pulmonary biopsy and pulmonary resection. Several instruments and alternatives have been used for thoracoscopy. Thoracoscopes are divided into rigid and flex-rigid/semi-rigid. Rigid scopes have been conventionally used, however literature suggests equal safety and efficacy of the flex-rigid scopes [2,3].

The thoracoscopy technique in relation to incision, point of entry, single versus double points of entry, anaesthesia utilised have been studied with various deviations as per the pulmonologists or surgeons and institute preferences. Minithoracoscopy defined as thoracoscopy with instruments of a diameter between 2 and $5 \mathrm{~mm}$ has been described by Marchetti et al. as complementary to the standard technique requiring however two separate small points of entry $[4,5]$. The same group have improvised and broadened the horizon of thoracoscopy in absence of pleural effusions with ultrasound guidance of the echocardiographic sliding sign without inducing a pneumothorax [6]; if this procedure has been recently underlined, it is an old procedure developed by several authors as Boutin, Tschopp, Noppen and others in patients with pleural tumours without pleural effusions or very few pleural fluid and also, and in particular, for sympatholysis (mainly for the treatment of hyperhidrosis). It was before the era of chest ultrasonography (CUS) and the artificial pneumothorax, which is the main point in this situation, was usually induced the day before the procedure to check the feasibility of the procedure. Thanks to the CUS and the presence of the 'sliding sign' making this step safer. In our case we describe the novel type of deviation for thoracoscopy in absence of pleural effusion. This procedure differed from that described by Marchetti et al. as we used a zero-degree camera lens with the thoracoport and single point of entry with induction of iatrogenic pneumothorax. The use of general versus local anaesthesia has been debated in literature. Use of only intrapleural anaesthesia has also been studied [7]. While most procedures are described under general anaesthesia, our technique deviated here in using local anaesthesia with moderate sedation.

\section{Conclusions}

This modified technique of dry thoracoscopy using zerodegree camera lens with the thoracoport using local anaesthetic agents with moderate sedation can be effectively used to manage such cases on a day care basis with early patient recovery and early post procedure discharge.

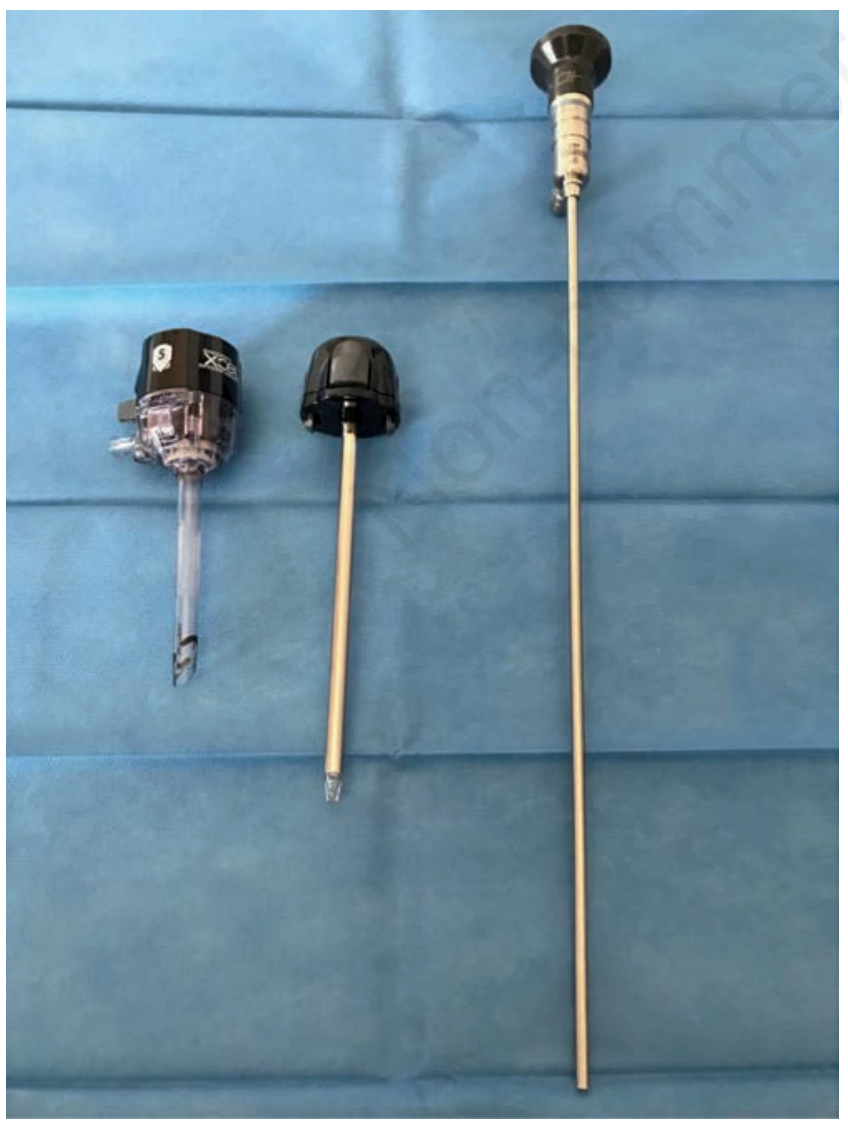

Figure 2. Image of the parts of thoracoport (left and centre tools) and the camera lens (right tool).

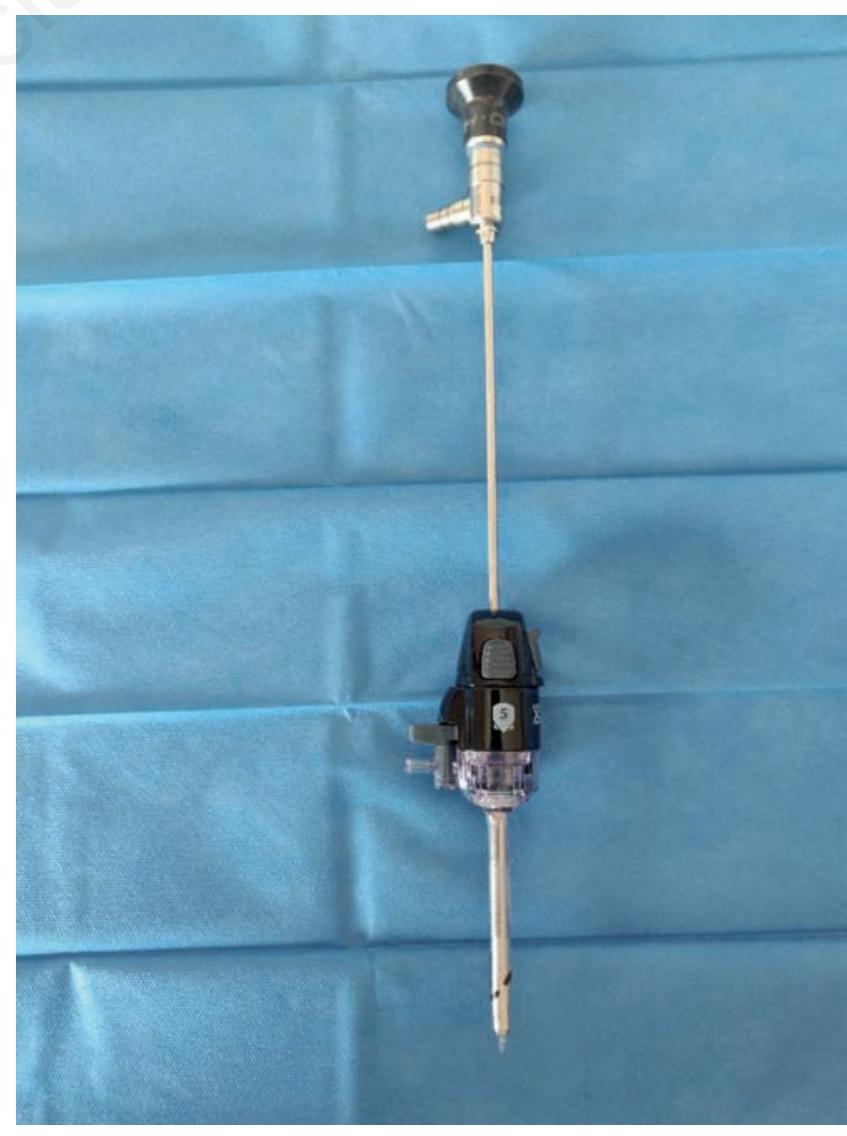

Figure 3. Assembly of thoracoport and camera lens ready for procedure. 


\section{References}

1. Braimbridge MV. The history of thoracoscopic surgery. Ann Thorac Surg 1993;56:610-4.

2. Rodriguez-Panadero F, Janssen JP, Astoul P. Thoracoscopy: general overview and place in the diagnosis and management of pleural effusion. Eur Respir J 2006;28:409-21.

3. Alraiyes AH, Dhillon SS, Harris K, Kaphle, U, Kheir F. Medical thoracoscopy: Technique and application. Pleura 2016;3:1-11.

4. Tassi G, Marchetti G. Minithoracoscopy. A less invasive approach to thoracoscopy. Chest 2003;124:1975-7.

5. Tassi G, Marchetti G, Pinelli V. Minithoracoscopy: a complementary technique for medical thoracoscopy. Respiration 2011;82:204-6.

6. Marchetti G, Valsecchi A, Indellicati D, et al. Ultrasound-guided medical thoracoscopy in the absence of pleural effusion. Chest 2015;147:1008- 12.

7. Mahmoud Reda, Asmaa Gomaa, Maged Hassan, et al. Intrapleural anaesthesia for medical thoracoscopy - a pilot study. Eur Respir J 2018;52:OA497. 